Andrea Baldo ${ }^{1}$, Gabriele Crimi ${ }^{2}$, Stefano De Servi ${ }^{1},{ }^{3}$, Maurizio Ferrario ${ }^{2}$, Luigi Oltrona Visconti ${ }^{2}$

${ }^{1}$ University of Pavia, Italy

2Division of Cardiology, Fondazione IRCCS Policlinico San Matteo, Pavia, Italy

${ }^{3}$ IRCCS Multimedica, Sesto San Giovanni, Milano, Italy

\title{
Neoatherosclerosis and plaque rupture as triggers for very late stent thrombosis in a bare-metal stent
}

\author{
Corresponding author: \\ Gabriele Crimi, MD \\ SC. Cardiologia, Fondazione IRCCS \\ Policlinico San Matteo \\ Piazzale Golgi 1 , \\ 27100 Pavia, Italy \\ Phone +390382 501598 \\ Fax +390382 \\ E-mail: gabrielecrimi@gmail.com \\ Medical Research Journal 2017; \\ Volume 2, Number 1, 34-35 \\ 10.5603/MRJ.2017.0006 \\ Copyright (C) 2017 Via Medica \\ ISSN 2451-2591
}

\begin{abstract}
Very late stent thrombosis has been increasingly described in the drug eluting stent era. A 56-year-old male presented with anterior ST-elevation myocardial infarction, he received a bare-metal stent in the left anterior descending artery in 2010. We obtained an optical coherence tomography scan, showing ruptured neoatherosclerotic plaque with thrombus.

Key words: stent thrombosis, optical coherence tomography, myocardial infarction
\end{abstract}

Med Res J 2017; 2 (1): 34-35
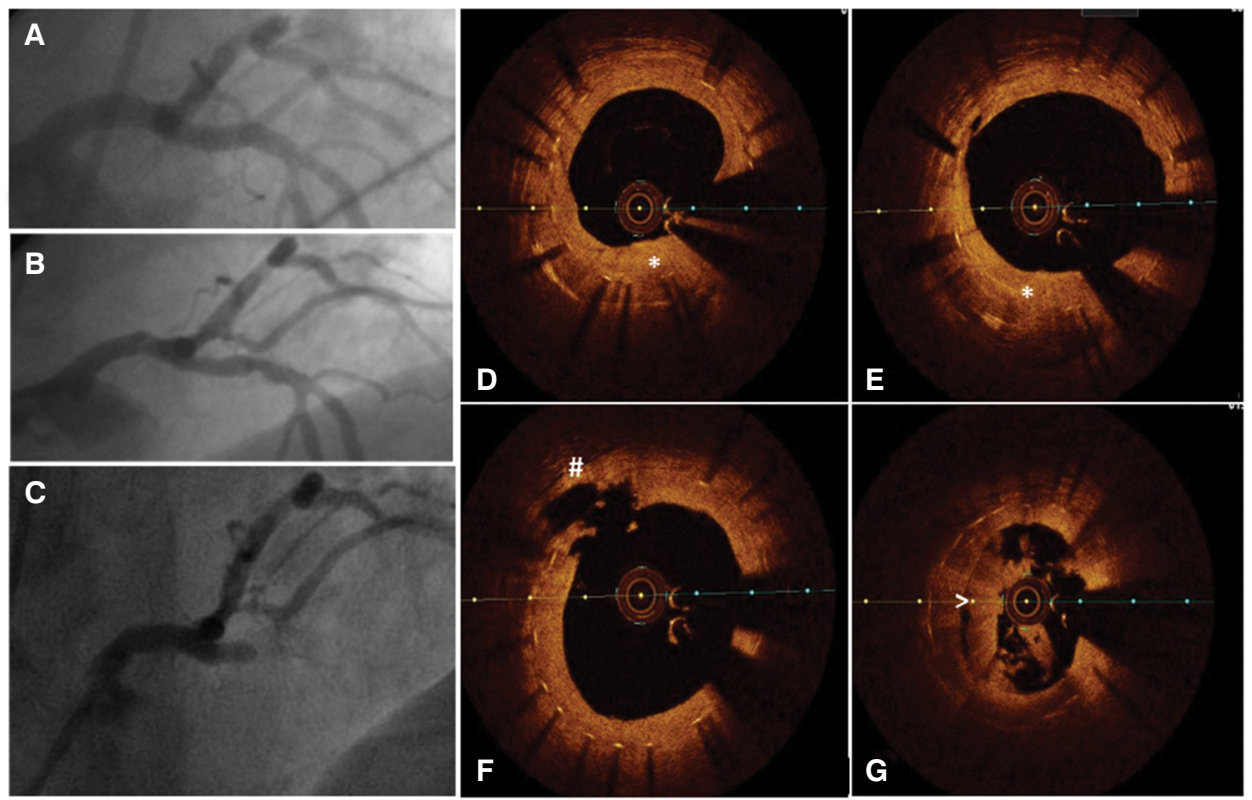

Figure 1. Neo-atherosclerosis following bare-metal stent (BMS) implantation complicated by very late stent thrombosis. A BMS was implanted in proximal left anterior descending (LAD) artery of a 56-year-old man during a ST-elevation myocardial infarction (STEMI) (A). After 2 year an eccentric neo-atherosclerotic plaque was documented (B). After 6 years, He was admitted for anterior STEMI (C). Optical coherence tomography (OCT) revealed the presence of complicated neo-atherosclerosis, with a glittering neointima along with dark areas, consistent with lipid pools (D-E, *), a ruptured and ulcerated plaque $(\mathbf{F}, \#)$, associated with an intracoronary thrombus $(\mathbf{G},>)$ 
Very late (beyond 1 year) stent thrombosis (VLST) has been increasingly described in the drug-eluting stent (DES) era [1].

A 56-year-old male presented to our with anterior ST-segment elevation myocardial infarction. He had underwent implantation of a bare-metal stent (BMS) $(3.5 \times 18 \mathrm{~mm})$ to the proximal left anterior descending artery in 2010 (Fig. 1A). A coronary angiogram in 2012 showed non-significant restenosis (Fig. 1B). We re-opened the occluded artery with a balloon and obtained an optical coherence tomography (OCT) scan. Intravascular imaging showed a ruptured plaque (Fig. 1F) with a thrombus (Fig. 1G), within complete neo-intimal coverage (Fig. 1D and E). We successfully implanted a DES $(3.5 \times 20 \mathrm{~mm})$ within the culprit lesion.

This case illustrates a rare case of VLST after BMS stenting and provides a unique insight into our knowl- edge regarding its pathophysiology. In summary, VLST may be found both in DES and in BMS. In patients with VLST, optimal intervention should be tailored to the underlying mechanism and OCT imaging should be considered in those cases.

\section{Acknowledgements}

The authors have no relationship with industries to disclose regarding the submitted article.

\section{Reference}

1. Windecker S, Meier B. Late coronary stent thrombosis. Circulation. 2007; 116(17): 1952-1965, doi: 10.1161/CIRCULATIONAHA.106.683995, indexed in Pubmed: 17965406. 